FORMATION Formation emploi

Revue française de sciences sociales

102 | avril-juin 2008

Pêle-mêle

\title{
Présentation de l'ouvrage Travail et reconnaissance des compétences
}

Bruno Lamotte

\section{OpenEdition}

1 Journals

Édition électronique

URL : http://journals.openedition.org/formationemploi/2310

DOI : 10.4000/formationemploi.2310

ISSN : 2107-0946

Éditeur

La Documentation française

Édition imprimée

Date de publication : 1 juin 2008

Pagination : 85-89

ISSN : 0759-6340

\section{Référence électronique}

Bruno Lamotte, «Présentation de l'ouvrage Travail et reconnaissance des compétences », Formation emploi [En ligne], 102 I avril-juin 2008, mis en ligne le 02 décembre 2009, consulté le 30 octobre 2020 URL : http://journals.openedition.org/formationemploi/2310 ; DOI : https://doi.org/10.4000/

formationemploi.2310

(c) Tous droits réservés 


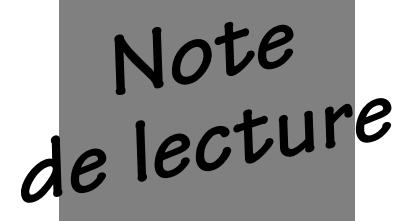

\section{Présentation de l'ouvrage "Travail et reconnaissance des compétences »}

\author{
Par Bruno Lamotte*
}

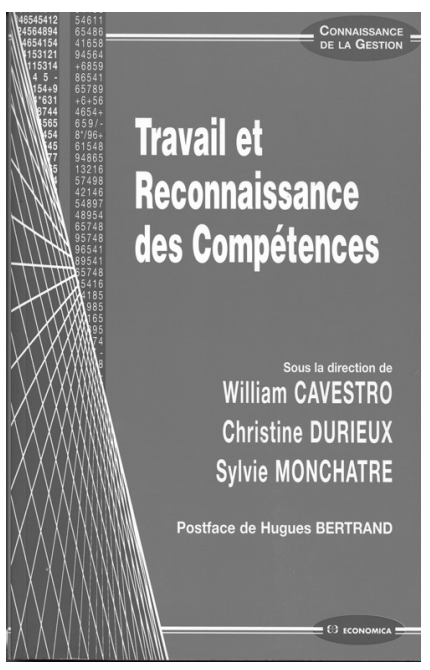

L'ouvrage, réalisé dans le cadre du travail du groupe compétences du Céreq, traite d'une évolution importante des modes de gestion contemporains de la main-d'œuvre, l'émergence de la gestion par les compétences. Plus exactement, il s'attaque à deux questions centrales, celle de la construction de la compétence à travers la formation, l'expérience professionnelle, la participation au collectif de travail et celle de sa reconnaissance dans les évaluations des entreprises, les conventions collectives et les mobilités professionnelles. Il porte sur trois niveaux d'analyse, celui de la relation entre la compétence individuelle et la compétence collective, celui de l'articulation entre le métier de l'individu et le métier de l'entreprise et celui des formes institutionnelles de reconnaissance dans les classifications de branche.

La première partie porte sur le lien entre la compétence individuelle et la compétence collective. Le premier chapitre, rédigé par Cavestro, Colin et
Grasser, souligne que la gestion des compétences dans les entreprises est une gestion individuelle, assez rétive à se situer sur le plan du fonctionnement du collectif de travail. Il est pourtant possible d'apparenter la compétence collective aux routines mises en avant par les économistes évolutionnistes pour expliquer le fonctionnement des organisations. Les compétences collectives surgissent dans des groupes hétérogènes engagés dans des actions communes et servent de cadre à l'action. À ce titre, la compétence collective apparaît comme le résultat d'un apprentissage d'équipe et entre en tension avec une démarche individuelle de gestion des compétences. Les référentiels de compétence jouent ici un rôle de pivot entre l'individuel et le collectif parce qu'ils introduisent la dimension du comportement à l'intérieur du

* Bruno Lamotte est économiste à l'université de Grenoble, LEPII (Laboratoire d'économie de la production et de l'intégration internationale)/ UMR-CNRS 5252. II a notamment publié : Abattu C., Lamotte B. (coord.) (2006), Diversité et inégalités: quelles pratiques de formation?, Paris, L'Harmattan, 268 p. Abattu C., Lamotte B. (2008), "Le développement de la formation des seniors: lutter contre les discriminations ou promouvoir la diversité ? ", in Roult N. (dir), Gérer les âges. Les petits pas dans les grandes entreprises, Éditions de l'ANACT (à paraître). 
collectif de travail dans l'évaluation des compétences individuelles. Leur rôle est de prendre en compte les relations de travail dans l'équipe et dans le management des personnes, la contribution individuelle au collectif est mise en valeur. In fine, c'est l'individu qui gère la tension entre participation au collectif et caractère personnalisé de la reconnaissance de la compétence. On pourrait en somme parler d'une reconnaissance faible et ambiguë de la dimension collective dans la gestion des compétences. Le deuxième chapitre rend compte d'une lecture croisée entre une sociologue et une ergonome sur le lien entre compétence individuelle et collective (Caroly et Cholez). En sociologie, et depuis Friedmann, les savoirs au travail ont un caractère social, quant en ergonomie on pose traditionnellement la question de l'adaptation du travail à l'homme en insistant sur l'individu. Mais dans l'histoire des deux disciplines, la sociologie rencontre l'hétérogénéité des individus au travail dans l'analyse des modalités d'émergence des compétences quand l'ergonomie, en cherchant à modifier les situations de travail avec les acteurs concernés, rencontre les modes de régulation collective des situations de travail. La modification des règles, la représentation et l'anticipation de l'action dépendent des relations avec les autres membres du groupe. Pour les auteurs, un point de rencontre fructueux entre une approche sociologique et une approche ergonomique portera sur l'analyse des controverses dans les groupes de travail. La notion de controverse rend compte de débats sur la façon de faire le travail au sein du collectif. La controverse joue un rôle dans la construction du groupe, ce qui intéresse la sociologie, et dans le développement de la compétence de l'individu, ce qui intéresse l'ergonomie.

Richebé, dans le troisième chapitre, approfondit la question de la fabrique des compétences collectives. Si depuis Taylor on sait que les compétences collectives sont plus que les compétences individuelles par effet d'organisation, l'approche taylorienne a longuement omis de reconnaître et d'analyser le niveau intermédiaire des collectifs de travail. Elle privilégie en effet la prescription du travail de l'exécutant à partir du point de vue de la direction, sans reconnaître le travail en équipe. Or la détention de compétences légitime une autorité technique et sociale dans le groupe. De plus, la compétence dans le travail est tributaire d'une dotation en ressources (moyens techniques, information...). La continuité entre dimensions individuelles et collectives est ainsi très forte et l'auteur suggère de s'appuyer sur l'économie des conventions pour mieux introduire le rôle des règles dans l'analyse des compétences : un individu qui suit des règles bénéficie de l'expérience cumulée de nombreux individus, résumée dans des règles.

La deuxième partie explore l'articulation entre compétences et métiers. Ces derniers sont compris dans le sens de Naville à la fois comme un attribut de l'activité individuelle (le métier est l'ensemble des capacités techniques de travail) et de l'activité d'une entreprise ou d'un secteur (le métier de l'entreprise). Dans un premier chapitre, Monchatre relève que les notions de métier et de compétence sont relativement antinomiques en sociologie des professions ou du travail. La notion de mandat peut alors servir à comprendre la façon dont une organisation reconnaît des métiers tout en les inscrivant dans une logique de contribution à sa propre performance. Pour l'auteur, la notion de mandat désigne une situation dans laquelle un donneur d'ordre détermine un résultat attendu, mais elle ne se réduit pas à cette seule dimension. Le mandat est le produit d'une construction sociale dans laquelle les collectifs mandataires interviennent. La pratique du mandat dans les fonctions de réalisation relève d'un recul de la prescription du travail et d'une recherche de salariés plus autonomes au lieu de simples exécutants. Ces mandats évoluent nécessairement avec l'environnement et le positionnement du mandant. Les collectifs de travail sont confrontés au caractère évolutif des mandats; de plus, le mandat introduit des mécanismes d'évaluation de la compétence partiellement externes au jugement des pairs dans la communauté de métier. Pour Monchatre, il y a lieu de différencier ainsi plus nettement une composante interne de la compétence et une composante externe, ce qui rapproche des notions anglo-saxonnes contenues dans les termes skills et competency.

Ughetto, dans un deuxième chapitre, explore les nuances de l'approche du métier pour l'entreprise et pour le salarié. Pour les entreprises, la réflexion sur le métier a indissociablement un caractère identitaire et stratégique : comment se positionner sur le marché 
dans le cœur de métier de l'entreprise, de façon à être reconnu comme une firme compétente ? C'est dans cette problématique que les démarches compétences, au sein des entreprises, trouvent leur sens. Il s'agit d'identifier les compétences pour les orienter vers les situations où on en aura le plus besoin. Le problème central est de gérer des mobilités qui ne reposent plus autant qu'autrefois sur la progression dans la maîtrise d'un métier, mais se construisent en lien avec l'entreprise elle-même.

Delignières, dans un troisième chapitre, approche les enjeux de la définition des compétences pour un secteur émergent. La téléphonie sanitaire et sociale se structure, au début des années 2000, en tentant de définir les compétences de l'écoute et un référentiel de compétences commun aux écoutants dans le secteur. Il en résulte la constitution des salariés comme acteur collectif et une meilleure identification des employeurs dans leur relation aux pouvoirs publics et au système de formation.

Vervaeke, dans le dernier chapitre, examine également la façon dont l'expertise dans le domaine du design a progressivement acquis une spécificité professionnelle et une autonomie à partir de trois segments, celui des salariés d'entreprises industrielles, celui des agences de conseil et celui des indépendants à forte notoriété. Un noyau stable de professionnels a constitué un champ disciplinaire autour de savoirs spécifiques, en contribuant à la formation des entrants notamment. Loin de s'opposer à la qualification, la compétence peut être considérée comme la manière dont la qualification, le métier sont concrètement mis en œuvre. Au total, dans cette partie, on montre que, par le jeu des mandats, les métiers sont en quelque sorte flexibilisés, mais certainement pas rejetés par l'approche compétence et qu'ils conservent toute leur importance dans la construction sociale des secteurs de l'économie.

La troisième partie peut alors poser le problème de la prise en compte des démarches compétences dans le système de relations professionnelles français. Un premier chapitre, de Baraldi et Durieux, pose les questions du rôle respectif de la régulation d'entreprise, de la régulation de branche et de l'articulation entre les deux. La logique de gestion par les compétences pourrait en première analyse être considérée comme une démarche de mise à l'écart des institutions externes à l'entreprise qui contribuent à valider les qualités du travail, en vue d'offrir des possibilités de construction d'un avantage compétitif de nature salarial. Mais selon les auteurs, bien des nuances doivent être apportées. Le rôle croissant de l'entreprise en matière de classification, autorisé par les grilles de classification en critères classants ${ }^{1}$, a été amplifié par les démarches compétences. Celles-ci en effet ne s'appuient que rarement sur la négociation d'entreprise, avant que la loi de 2005 ne place la Gestion prévisionnelle de l'emploi et des compétences dans le champ des négociations obligatoires ; elles ont alors le caractère de règles de gestion plus ou moins stables et partagées dans la hiérarchie de l'entreprise. Dans ce contexte, la négociation de branche, qui reste dynamique, demeure un point de repère important dans tous les secteurs. Les organisations syndicales y restent très attachées ; l'État favorise également l'activité conventionnelle de branche par plusieurs dispositifs. Aussi bien en matière de classification et de salaire qu'en matière de formation professionnelle, la branche structure une partie du dispositif des démarches compétences.

Oiry, dans le deuxième chapitre, approfondit l'analyse au sujet des positions des acteurs patronaux et syndicaux français. Il ne fait guère de doute que la gestion par les compétences est un thème amené initialement, dans le dialogue social français, par le Mouvement des entreprises de France (MEDEF), qui $\mathrm{y}$ voit une réponse nécessaire aux changements techniques et à la mondialisation de la concurrence économique. Les organisations syndicales détiennent cependant, dans l'histoire de leurs débats internes, beaucoup d'éléments permettant de se positionner. Surtout à la Confédération générale du travail (CGT) et à la Confédération française démocratique du travail (CFDT), le débat va être assez dense et conduire à des prises de position nuancées. À l'instar de la Confédération générale des cadres (CFE-CGC), la réflexion ira souvent au-delà du sujet proprement dit et portera sur les principes de fonctionnement du

\footnotetext{
${ }^{1}$ Dans les années 70, les grilles de classification des conventions collectives ont beaucoup évolué. Le principe d'une description précise des postes a reculé au profit de grilles fixant, dans des termes plus généraux, les critères d'évaluation retenus tels que l'autonomie ou le niveau de formation requis, ce qui laisse plus de place à interprétation.
} 
système de relations professionnelles. En entrant dans la démarche compétences, faut-il entrer dans la participation à la construction des instruments de gestion des entreprises? Pour Oiry, c'est une question-clé à laquelle sont confrontées les organisations syndicales.

Dans un troisième chapitre, Bagorsky et Brochier analysent la réforme de la formation issue de l'accord national interprofessionnel de 2003, au prisme de la négociation des compétences entre l'individu et l'entreprise. Dans l'apparition du droit individuel à la formation (DIF) ou dans la nouvelle catégorisation des actions du plan de formation, de nouvelles prises en compte de la problématique des compétences des salariés ont été introduites par les négociateurs, même si la trace en est diluée et si les grandes entreprises y verront plus d'apports que les petites. Les salariés pourraient y retrouver plus d'autonomie et de prise sur l'évolution de leur propre compétence.

Le quatrième chapitre, de Lefresne, ouvre sur le caractère européen d'une prise en compte de la qualification comme un processus et non comme un ensemble clos de qualités requises. Le thème de la formation tout au long de la vie rend compte d'une problématique des compétences qui traverse les pays européens, la recherche d'une organisation du travail plus réactive et la gestion d'une initiative plus étendue du salarié. Il reste que les systèmes de formation initiale et continue conservent un caractère intrinsèquement national. Les pays d'Europe du Nord, d'Europe continentale, le Royaume-Uni présentent des articulations entre formation professionnelle initiale et continue, des systèmes de relations professionnelles aux fonctionnements très différents. Les réponses à la problématique des compétences sont également différenciées, mais l'auteur montre qu'existe un tronc commun de problèmes autour de la question de la nature des droits sociaux accordés aux personnes mobiles, souvent menacées de précarité, et autour de la question de l'égalité de ces droits par rapport aux travailleurs plus stabilisés dans leurs carrières professionnelles.

L'ouvrage est clôturé par une postface de Hugues Bertrand. Il attire notamment l'attention sur la portée profonde des démarches compétences en établissant un parallèle entre les normes tayloriennes qui visaient l'économie des moyens de production et les outils de gestion des compétences qui visent eux à l'économie des savoirs et des connaissances. Il s'agit, selon lui, de changements profonds mais risqués, car ces démarches peuvent glisser vers le simple tri des meilleurs, ce qui constituerait un contresens au regard de la recherche d'efficacité collective initiale. La façon dont le système de relations professionnelles traitera de la démarche compétences est alors une question centrale pour limiter les risques d'une évolution dangereuse.

Pour conclure, soulignons l'avancée que représente ce livre dans la compréhension des dimensions individuelles et collectives des compétences. Des questions bien sûr restent ouvertes ou à approfondir. On peut notamment évoquer celle des conséquences sociales de la démarche compétences. Une partie de la littérature sur les compétences s'inquiète de la remise en cause des statuts et des positions des salariés qu'elle peut amener. L'introduction de l'ouvrage rappelle la démarche des années 80 , qui visait à moderniser la production sans exclure les jeunes sans qualification et les salariés les plus fragiles. Ce champ de préoccupations ne trouve pas beaucoup d'échos dans la suite de l'ouvrage. Pourtant, il est souligné que la démarche compétences est conçue par le patronat comme une réponse logique à des techniques de production sollicitant plus de savoirs et à un contexte concurrentiel générant des changements et des adaptations rapides. Dans la situation de chômage élevé, où de nombreux jeunes quittent le système éducatif sans qualification professionnelle aboutie, où de nombreux seniors à faible formation initiale ont peu bénéficié de la formation tout au long de la vie, les démarches compétences ont-elles une capacité d'inclusion de la main-d'œuvre la moins qualifiée? En un sens, les démarches de Gestion prévisionnelle des compétences peuvent assurer cette fonction et préparer les évolutions nécessaires dans une entreprise, un territoire ou un secteur. Mais on peut craindre que, dans certains cas, une gestion des compétences ne risque d'éliminer les plus fragiles et de reporter hors de l'entreprise la prise en charge des « moins compétents », ou supposés tels. La question est en filigrane de plusieurs contributions; il serait intéressant qu'elle revienne explicitement dans le 
débat pour deux raisons. En premier lieu, le collectif de travail peut d'abord être compris comme une communauté plus vaste que celle de l'entreprise ou $\mathrm{du}$ secteur, c'est l'idée du travailleur collectif, abstrait et général, relativement indépendant de sa structuration professionnelle et sectorielle. Comment le monde des ouvriers ou celui des employés viventils la démarche compétences? En deuxième lieu, le dialogue social sur la gestion des compétences, on l'a dit, est en phase de démarrage sur les questions de Formation tout au long de la vie et de Gestion prévisionnelle des compétences. Les organisations syndicales devraient, chacune selon sa propre approche, se positionner sur cette question. Même avec l'entrée dans une logique plus gestionnaire, comme l'évoque l'ouvrage sous forme de question, les risques de la démarche compétences devraient certainement rester un élément de leurs préoccupations.

\section{Référence de l'ouvrage}

Cavestro W., Durieux C. et Monchatre S. (dir.) (2007),

Travail et reconnaissance des compétences, Économica, $224 \mathrm{p}$. 